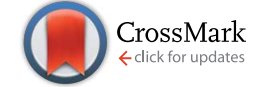

Cite this: RSC Adv., 2017, 7, 7502

Received 28th October 2016 Accepted 3rd January 2017

DOI: 10.1039/c6ra25991j

www.rsc.org/advances

\section{Separation of cyanide from an aqueous solution using armchair silicon carbide nanotubes: insights from molecular dynamics simulations}

\begin{abstract}
Alireza Khataee, ${ }^{*}$ Golchehreh Bayat and Jafar Azamat
Separation of cyanide, as a model contaminant, from aqueous solution was investigated using molecular dynamics simulations. In this research, cyanide separation was investigated using armchair silicon carbide (SiC) nanotubes of four different diameters at different applied pressures. The systems included $(5,5)$, $(6,6),(7,7)$ and $(8,8) \mathrm{SiC}$ nanotubes placed between two graphene sheets, and an external pressure was applied to the system. The ion permeability, the radial distribution function of nanotube-water and ionwater, the retention time of the cyanide ions, the density of water and water flux and the hydrogen bonding between inner water molecules were investigated. The results showed that all four studied nanotubes accepted water molecules into their interiors, and the $(5,5) \mathrm{SiC}$ nanotube could provide high rejection $(100 \%)$ of cyanide ions.
\end{abstract}

\section{Introduction}

Cyanide is a damaging and poisonous pollutant, which threatens the environment and human health. Cyanide is widely used in diverse industries including electroplating, mining, photoprocessing, fertilizer production, gas production, pharmaceuticals and plating. ${ }^{1}$ The existence of cyanides in wastewater poses a high risk and hence, it should be removed from wastewaters before release. ${ }^{2}$ Cyanides cause extensive fish death and affect the microscopic and invertebrate organisms in water. It is also toxic and damaging for human health, causes neurological effects (quick breathing, tremors), weight loss, nerve injury and disease. Skin contact with cyanides may produce irritation and sores. ${ }^{3-5}$

There are different ways of treating cyanide-containing wastewaters, including chemical oxidation, physical methods and biological processes. ${ }^{6-10}$ For example, Simsek et al. conducted column and batch studies with a Purolite resin in order to remove cyanide ions from aqueous solutions. ${ }^{1}$ Moreover, Hijosa-Valsero et al. tested plasma discharge technology for cyanide removal from water with an initial concentration of 1 $\mathrm{mg} \mathrm{L}^{-1} \cdot{ }^{11}$ They proposed a coaxial DBD plasma reactor only for the treatment of dilute solutions such as drinking water. In another study, Sarla and coworkers studied the oxidation process of cyanide in aqueous solutions with $\mathrm{H}_{2} \mathrm{O}_{2}$ as the oxidant and $\mathrm{Cu}^{2+}$ as the catalyst. They found that chemical oxidation by $\mathrm{H}_{2} \mathrm{O}_{2}$ is slow, whereas by $\mathrm{UV} / \mathrm{H}_{2} \mathrm{O}_{2}$ it is much faster,

Research Laboratory of Advanced Water and Wastewater Treatment Processes, Department of Applied Chemistry, Faculty of Chemistry, University of Tabriz, 51666-16471 Tabriz, Iran. E-mail: a_khataee@tabrizu.ac.ir; ar_khataee@yahoo. com; Fax: +98 4133340191; Tel: +98 4133393165 and in both cases, $\mathrm{Cu}^{2+}$ accelerated the oxidation process, acting as a catalyst. ${ }^{12}$ In a similar study, the oxidation process of free cyanide with $\mathrm{H}_{2} \mathrm{O}_{2}$ catalyzed by activated alumina was studied by Chergui et al. They claimed that their proposed method had advantages over the method based on copper catalyst. ${ }^{13}$

Akcil et al. investigated the biological degradation of cyanide by specific bacteria. These bacteria could efficiently degrade cyanide into less toxic compounds, namely ammonia and carbonate..$^{14}$ In addition, various biodegradation methods for cyanide removal were introduced in a review by Ibrahim et al. ${ }^{15}$

Some of these methods are not economical or environmentally friendly because of the application of dangerous chemical reagents. Moreover, they produce poisonous residues and cannot completely reduce all cyanide complexes. ${ }^{1,16}$

In this regard, for water purification and to meet qualification requirements, nanotechnology has emerged as a novel and powerful tool. ${ }^{17,18}$ Nanotubes are an excellent and appropriate choice as ion selective materials. ${ }^{19-21}$ Although carbon nanotubes have received more attention, ${ }^{22,23}$ other materials, such as boron nitride (BN) ${ }^{24-26}$ and silicon carbide (SiC), ${ }^{27}$ can also be used to fabricate nanotubes. SiC nanotubes were first synthesized in $2001 .^{28}$ They exhibit better reactivity than carbon nanotubes due to their polar nature. ${ }^{29}$ For example, theoretical studies have shown that SiC nanotubes are an excellent material for hydrogen storage. ${ }^{30}$ According to these studies, $\mathrm{SiC}$ nanotubes exhibit a $\mathrm{H}_{2}$ binding energy that is $20 \%$ greater than that of carbon nanotubes. In another study, the capability of $\mathrm{SiC}$ nanotubes as a sensor for formaldehyde detection was demonstrated. $\mathrm{CH}_{2} \mathrm{O}$ molecules were chemisorbed to $\mathrm{SiC}$ nanotubes with a more noticeable adsorption energy, relative to that of carbon nanotubes. Zhao et al. studied the catalytic behavior of SiC 
nanotubes. Their theoretical calculations showed that $\mathrm{SiC}$ nanotubes can efficiently break the $\mathrm{N}-\mathrm{H}$ bond of ammonia and the $\mathrm{O}-\mathrm{H}$ bond of $\mathrm{H}-\mathrm{OX}\left(\mathrm{X}=\mathrm{H}, \mathrm{CH}_{3}\right.$, and $\left.\mathrm{C}_{2} \mathrm{H}_{5}\right) \cdot{ }^{31}$ Moreover, some researchers have shown the ion selectivity of $\mathrm{SiC}$ nanotubes. $^{21,32}$ In our previous study, separation of nitrate ions was studied using armchair SiC nanotubes. We found that $(8,8) \mathrm{SiC}$ nanotubes can remove nitrate ions from aqueous solutions. ${ }^{32}$ In a similar study, Hilder and coworkers demonstrated the ionselectivity of $(6,6)$ and $(7,7) \mathrm{SiC}$ nanotubes. They found that no sodium ions were able to pass through the nanotubes, whereas chloride ions were able to pass. ${ }^{21}$

Fluid-particle dynamics simulations consist of computational methods for macroscale (smoothed particle hydrodynamics (SPH)), ${ }^{33}$ mesoscale (dissipative particle dynamics (DPD)), ${ }^{34}$ and microscale (molecular dynamics (MD)) systems. ${ }^{32}$ In the field of computational chemistry, MD simulation is an invaluable method for studying the atom flow through nanopores. $^{35,36}$ The atom resolution can be simulated accurately via $\mathrm{MD}$, and therefore it is possible to investigate the transport characteristics because of the tunable long timescale of $\mathrm{MD}$, which allows researchers to collect adequate statistics. Moreover, numerous permeation events for single ions and water molecules through nanopores can be studied via MD. ${ }^{37}$ Thus, in this study, the passage of cyanide ions through SiC nanotubes under pressure was studied via MD simulations.

\section{Simulation details}

Four types of armchair SiC nanotubes, including $(5,5),(6,6)$, $(7,7)$ and $(8,8)$ nanotubes with a length of $20 \AA$ were selected. The system domain contained an armchair $\mathrm{SiC}$ nanotube placed between two graphene sheets, potassium and cyanide ions, and water molecules. The concentration of dissolved potassium and cyanide ions was $0.3 \mathrm{M}$. The dimensions of the simulation box were $(x \times y \times z) 30 \times 30 \times 80 \AA^{3}$. The periodic boundary conditions were used in all three directions. The short-range Lennard-Jones interaction parameters are summarized in Table 1. Long-range electrostatic interactions were calculated using the PME (Particle Mesh Ewald) ${ }^{38}$ method with a 12 A cut-off for van der Waals interactions. The optimized geometries of these nanotubes were obtained at the B3LYP level of theory with the $6-31 \mathrm{G}^{* *}$ basis set using the GAMESS program. ${ }^{39}$ The value of 1.8 A was obtained for the optimized Si$\mathrm{C}$ bond length. This amount of bond length was used in other researchers, too. ${ }^{\mathbf{4 0 , 4 1}}$ For water molecules, the TIP3P water

Table 1 Lennard-Jones parameters

\begin{tabular}{llll}
\hline Atoms & $\begin{array}{l}\varepsilon \\
\left(\mathrm{kcal} \mathrm{mol}^{-1}\right)\end{array}$ & $\begin{array}{l}R_{\min } / 2 \\
(\AA)\end{array}$ & Ref. \\
\hline Silicon & 0.469 & 1.66 & 59 \\
Carbon (nanotube) & 0.086 & 1.52 & 59 \\
Carbon (graphene) & 0.086 & 1.91 & 60 \\
Potassium & 0.087 & 1.76 & 61 \\
Carbon (cyanide) & 0.105 & 1.93 & 62 \\
Nitrogen (cyanide) & 0.069 & 1.83 & 62
\end{tabular}

model $^{42}$ was used to exactly reproduce the entropic and hydrogen bonding behavior of water.

NAMD 2.10 (ref. 43) was used for performing MD simulations, similar to previous study, ${ }^{4-47}$ and visualized via VMD1.9.2. ${ }^{48}$ The van der Waals interaction energies $\left(E_{\mathrm{LJ}}\right)$ are defined by eqn (1) as follows: ${ }^{49}$

$$
E_{\mathrm{LJ}}=4 \sqrt{\varepsilon_{\mathrm{a}} \varepsilon_{\mathrm{b}}}\left[\left(\frac{\left(\sigma_{\mathrm{a}}+\sigma_{\mathrm{b}}\right)}{2 r_{\mathrm{ab}}}\right)^{12}-2\left(\frac{\left(\sigma_{\mathrm{a}}+\sigma_{\mathrm{b}}\right)}{2 r_{\mathrm{ab}}}\right)^{6}\right]+\frac{q_{\mathrm{a}} q_{\mathrm{b}}}{4 \pi \varepsilon_{0} r_{\mathrm{ab}}}
$$

where $\varepsilon_{\mathrm{a}}$ and $\sigma_{\mathrm{a}}$ are the Lennard-Jones parameters for atom a, $r_{\mathrm{ab}}$ is the distance between two atoms, and $q_{\mathrm{a}}$ and $q_{\mathrm{b}}$ are the partial charges associated to atoms. The armchair SiC nanotubes were placed between two graphene sheets that were fixed during the simulation. As indicated by Bucior et al. the flexibility of the nanotubes is important when the diameter of the nanotube and the size of the molecules are about the same. ${ }^{50}$ However, in this study, the molecule size was smaller than the pore size. Thus, fixing the nanotube did not affect the results. ${ }^{51}$

The simulation box was exposed to zero-temperature energy minimization for $40 \mathrm{ps}$, then equilibrated at $298 \mathrm{~K}$ for $10 \mathrm{ps}$ and finally, MD simulation was performed for $5 \mathrm{~ns}$. The temperature was fixed at $298 \mathrm{~K}$ using the Langevin dynamics method. External constant forces perpendicular to the cross-section of the nanotube were applied to the water molecules in a selected area of the system (within a distance of $9 \AA$ to the left or right boundary of the system).

The applied forces generate a pressure difference through the SiC nanotubes that forces the fluid to flow, which is known as pressure-driven flow. ${ }^{52,53}$ The applied force was defined as $F=$ $\triangle P A / n$, where $F$ is the force exerted to the chosen section of the box (in pN), $\Delta P$ is the pressure difference (in $\mathrm{Pa}$ ), $A$ is the cross sectional area of the system (in $\mathrm{m}^{2}$ ), and $n$ is the number of water molecules in the selected region (see Fig. 1). Pressure ranged from 10 to $200 \mathrm{MPa}$ and was applied to nearly 542 water molecules.

\section{Results and discussion}

In this study, single-walled $(5,5),(6,6),(7,7)$ and $(8,8) \mathrm{SiC}$ nanotubes with a length of $20 \AA$, fixed between two graphene

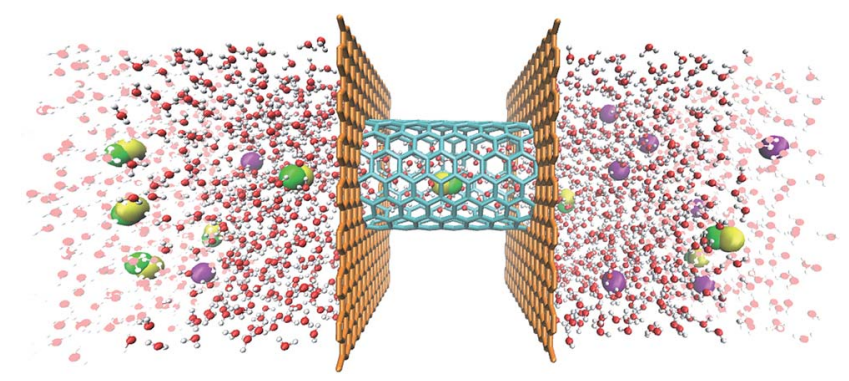

Fig. 1 The $(8,8) \mathrm{SiC}$ nanotube fixed between two graphene sheets. Two water reservoirs are affixed to each side of the box. In the glass region, external forces are applied to water molecules so as to create a pressure difference across the membrane (potassium: purple; nitrogen: green; carbon: yellow; oxygen: red; and hydrogen: white). 
sheets were studied for the separation of cyanide ions from a potassium cyanide (KCN) aqueous solution. In order to study the ion selectivity of this system, the following properties were evaluated: arrangement of the inner water molecules, number of hydrogen bonds between water molecules, ion retention time, density of the inner water, water flux, and radial distribution functions (RDF) of the water-nanotube and water-ion.

\subsection{Water conduction}

All four SiC nanotubes accepted water molecules into their interior. The arrangement of the water molecules inside the nanotubes changed on increasing the nanotube diameter. The arrangement of the water molecules, illustrated in Fig. 2, was verified by the RDF between inner water molecules and the nanotube (see Fig. 3). It was found that for the $(5,5) \mathrm{SiC}$ nanotube, at low pressure (10 MPa), inner water molecules formed a two-chain structure. This structure was similar to that formed inside the $(6,6) \mathrm{SiC}$ nanotube, and changed to a cylindrical arrangement at higher pressures. In the $(7,7) \mathrm{SiC}$ nanotube, inner water molecules were arranged into the form of a cylinder, with a single-file structure inside it. Finally, inside the $(8,8) \mathrm{SiC}$ nanotube, water molecules were arranged forming an structure with two concentric cylinders. RDFs between inner water molecules and the nanotube confirmed the arrangements of the water molecules. Likewise, Fig. 4 displays the RDF between the inner water molecules and the $(5,5) \mathrm{SiC}$ nanotube at the different studied pressures. In this figure, the differences in the water molecule arrangements between low and high pressure were evident. (a)
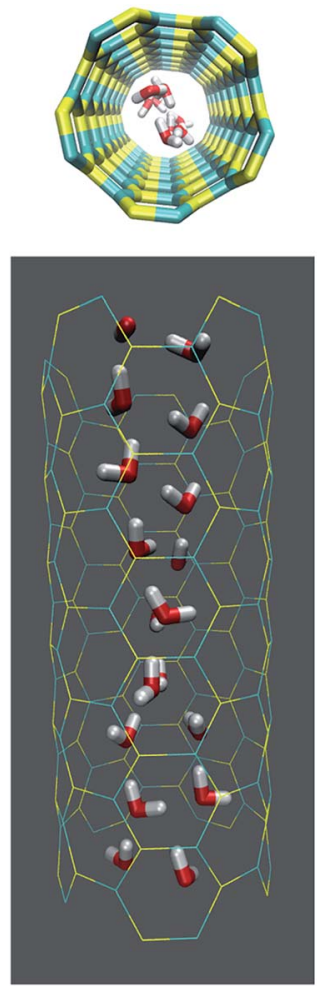

(b)
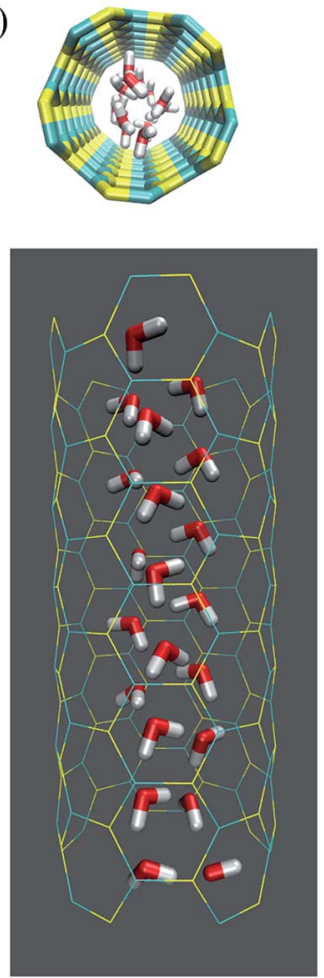

Fig. 2 Arrangement of the water molecules inside a $(5,5) \mathrm{SiC}$ nanotube under different pressures, namely (a) at $10 \mathrm{MPa}$ and (b) $25 \mathrm{MPa}$.
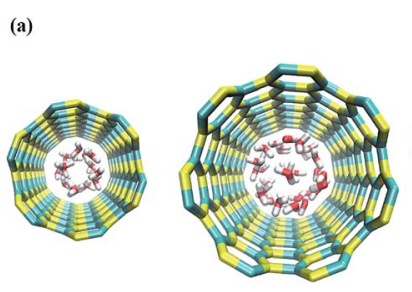

$(7,7)$

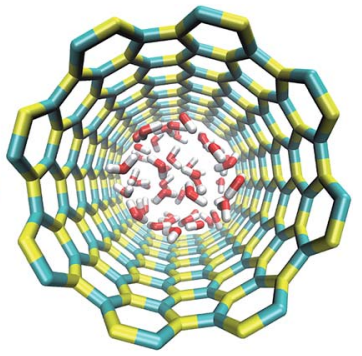

$(8,8)$

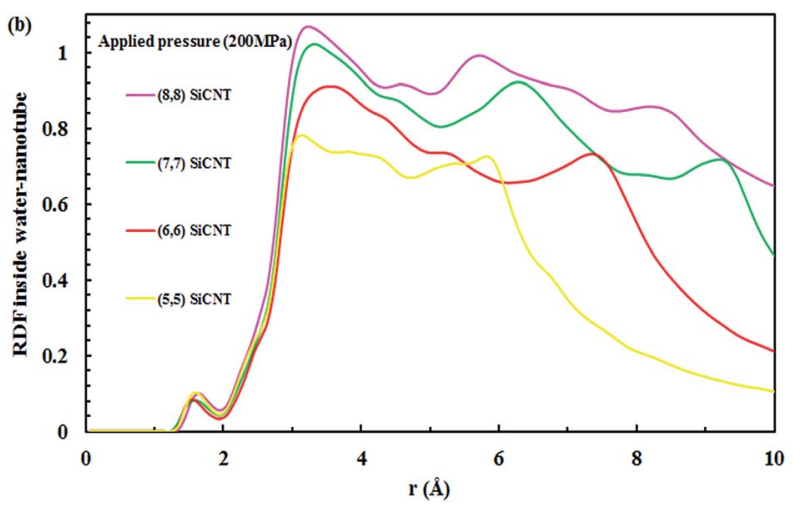

Fig. 3 (a) Water arrangement inside SiC nanotubes of different diameter and (b) RDF of inner water molecules-SiC nanotubes at 200 $\mathrm{MPa}$.

Fig. 5 displays the density of the inner water molecules in the four types of SiC nanotubes at the applied pressures during the 5 ns MD simulations. As it can be seen, the water density increased upon increasing the applied pressure because of the high transport of water molecules at high pressures. Water density also increased as the nanotube diameter became larger. The effect of nanotubes diameter on water density was stronger than that of applied pressure. Fig. 6 shows the water flux values through $(6,6)$ and $(8,8) \mathrm{SiC}$ nanotubes for the different applied

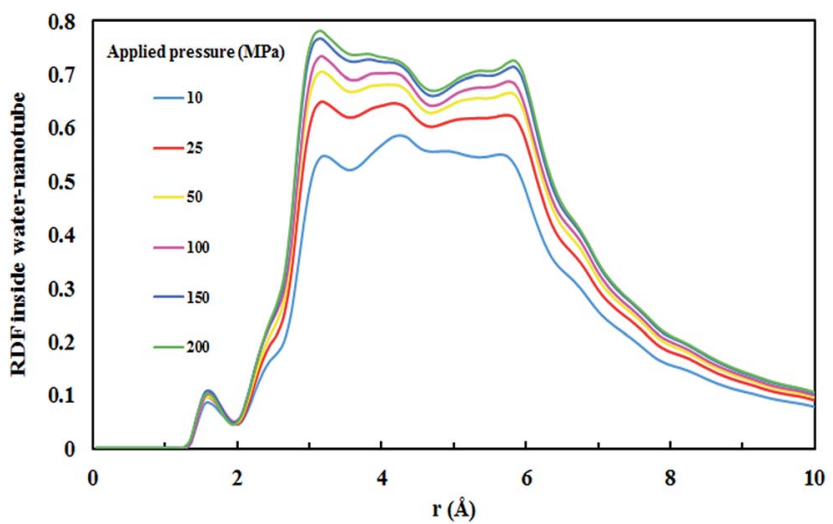

Fig. 4 RDF of inner water molecules-(5,5) SiC nanotube at different applied pressures. 


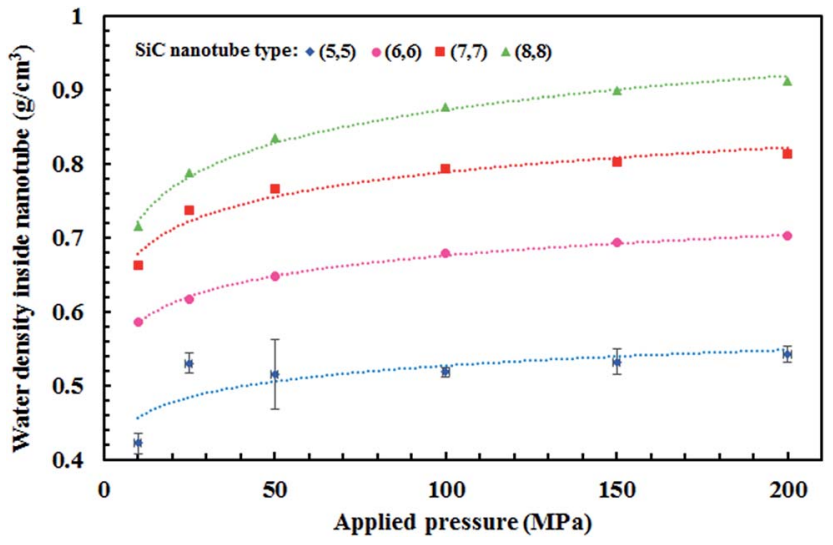

Fig. 5 Density of water molecules inside SiC nanotubes of different diameters and at different pressures. Error bars represent the uncertainty of the corresponding data and are only shown for values greater than the data points.
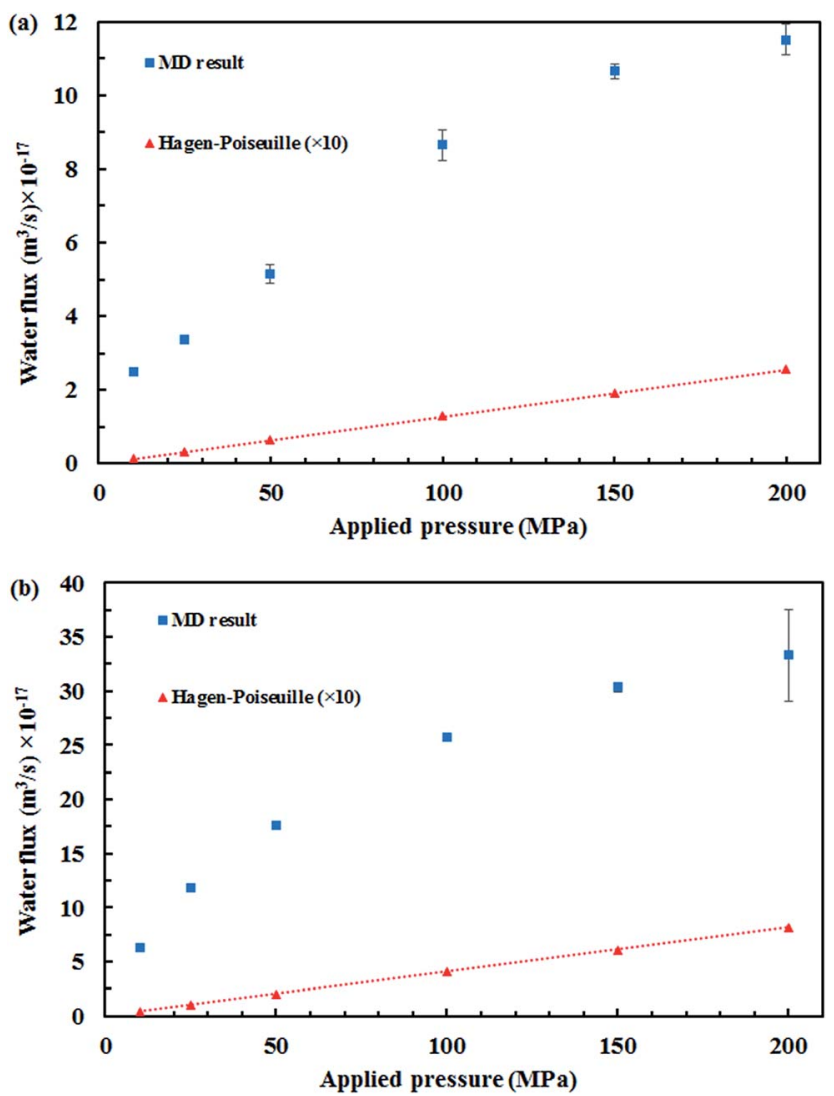

Fig. 6 Theoretical and simulated water flux through (a) $(6,6)$ and (b) $(8,8) \mathrm{SiC}$ nanotubes as a function of the applied pressure. Error bars represent uncertainty of the corresponding data and are only shown for values greater than the data points.

pressures. These values were obtained using MD simulations and the Hagen-Poiseuille (HP) equation as described below: ${ }^{54}$

$$
E_{\mathrm{LJ}} Q=\frac{D^{2}}{128\left(\frac{l}{A}\right) \mu} \Delta P
$$

where $Q$ is the water flux, $\mu$ is the dynamic viscosity, and $D, l$ and $A$ are the diameter, length and cross-section of the nanotube, respectively. This figure shows that water flux increased as the applied pressure increased. According to the HP equation, which is based on a macroscale continuum approach, the pressure increased linearly. This linear behaviour could also be seen according to eqn (2). However, the macroscale continuum assumption is not accurate for nanotubes. This is because of the no-slip boundary condition, which is only satisfied at a macroscopic scale, in which the length scale of the problem is much larger than the mean free path of the molecules. This effect is described by the Knudsen number (Kn), which can be defined $\mathrm{as}^{55} \mathrm{Kn}=\lambda / L$, where $\lambda$ is the molecular mean free path and $L$ is the characteristic length scale, which in this study is equal to the diameter of the nanotubes. The deviation of the HP calculation and MD simulation is also due to the molecular interactions, which are not important in the macroscale continuum assumption. The HP equation only considers viscosity as a physical property and disregards other molecular effects.

The water network structure inside the nanotubes led to the formation of hydrogen bonds. The number of water molecules that entered the nanotube was directly proportional to the applied pressure, i.e., the number of water molecules was higher at higher pressures. Thus, the number of hydrogen bonds also increased, as can be observed in Fig. 7.

\subsection{Ion transportation}

The number of cyanide ions that passed through the SiC nanotubes of various diameters under the applied pressure was counted during the simulation time to investigate the cyanide rejection ability. Indeed, for the passage of ions through the nanotube, ion size should be smaller than the diameter of the nanotube. Therefore, the water molecules that form the hydration shell of the cyanide ion should be removed. This dehydration requires a large amount of energy that is known as the energy barrier. The applied pressure to the system provided

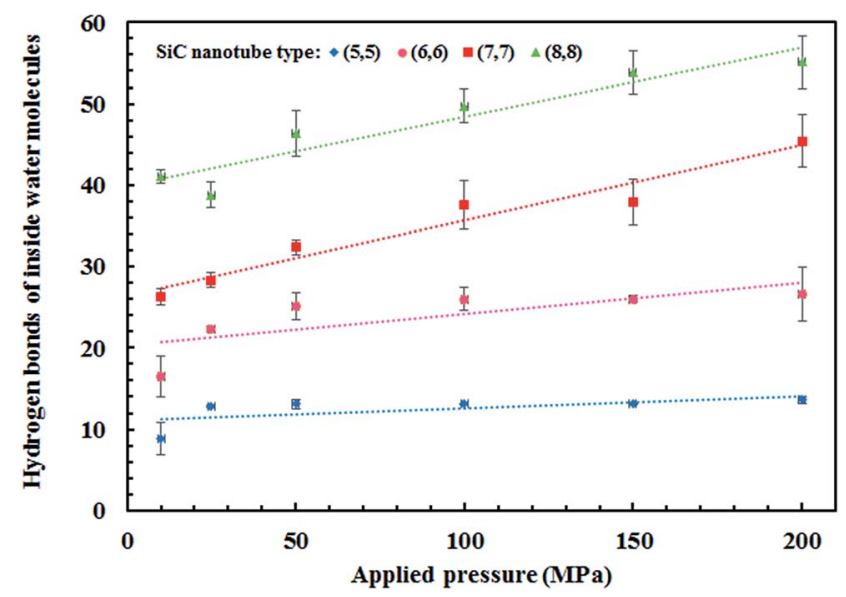

Fig. 7 Number of hydrogen bonds between the water molecules inside SiC nanotubes of different diameters. Error bars represent uncertainty of the corresponding data and are only shown for values greater than the data points. 
the required energy to overcome this barrier. Fig. 8 shows the effect of SiC nanotube diameter and applied pressure on cyanide ion rejection. As can be seen, rejection ability decreased when both the SiC nanotube diameter and applied pressure increased. The ion rejection rate decreased when increasing the pressure mainly because the large force applied on the ions helped to overcome the energy barrier. On the other hand, rejection rate was greater than $78 \%$ in the $(5,5)$ SiC nanotube $(100 \%$ at $10 \mathrm{MPa})$. This is due to the limited diameter of the $(5,5)$ SiC, which causes a high energy barrier for cyanide permeation at low pressure. Indeed, at low pressure, the applied force is not enough to separate water molecules from the ion hydration shell. SiC nanotubes with larger diameters allowed ions to pass through them more easily; subsequently, they had a lower rejection rate, with the rejection rate for the $(8,8) \mathrm{SiC}$ nanotube being $0 \%$. This was because the energy barrier for ions to enter larger nanotubes was lower.

Fig. 9 shows the pressure effect on the separation time of the ions. Cyanide ions separated from the aqueous solution at the beginning of the simulation time at higher pressures, whereas at lower pressures, it took a longer time for them to be removed from the aqueous solution. Pressure force exerted to the flowing fluid through a distance resulted in a kind of work called flow work, which is defined as follows: ${ }^{56}$

$$
e_{\text {flowwork }}=P / \rho
$$

where $\rho$ is the density of the fluid. As shown by eqn (3), at higher applied pressures to the system, the consumed energy is higher. Although removal time was small at higher pressure (Fig. 9), the amount of energy required for this was also high. Therefore, it is important to optimize the energy according to our needs.

Fig. 10 shows the $z$ position of the cyanide ions inside the $(8,8) \mathrm{SiC}$ nanotube during the simulations. The results did not show any overlap between the retention time of ions at high and low pressures. If the applied driving force to the system is a type of high energy (such as pressure) the ions traverse through the nanotube independently, i.e., the first ion does not need the assistance from the second ion to push it outwards through the

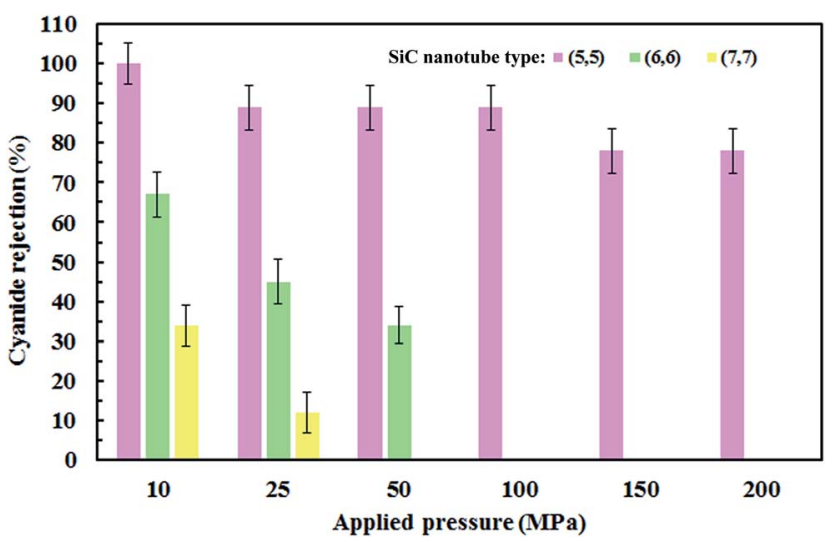

Fig. 8 Ion rejection percentages for different applied pressures (MPa) and $(\mathrm{SiC})$ nanotube diameters. Error bars represent uncertainty of the corresponding data.

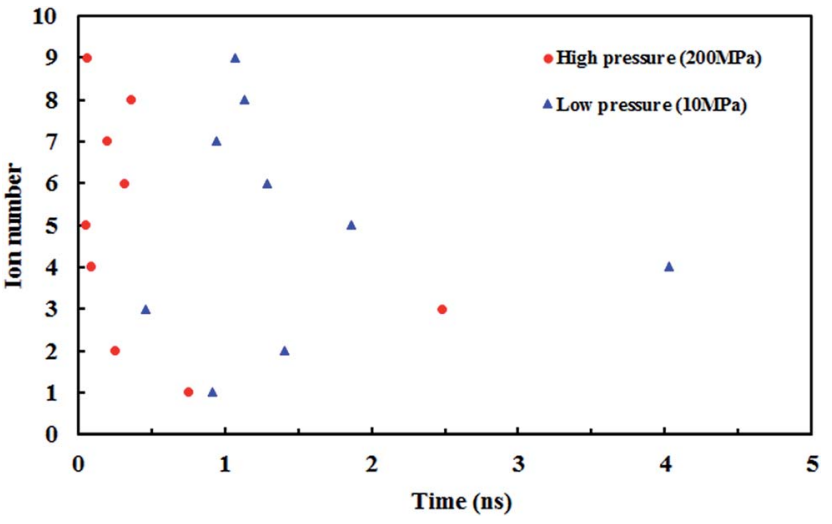

Fig. 9 Effect of low and high applied pressure on the permeation time of ions.

nanotube. On the other hand, if the applied driving force is smaller (such as a small electrical field) the first ion needs a second ion to help it to move outside the nanotube. ${ }^{32}$

Fig. 11 illustrates the effect of the applied pressure on the retention time of the ions (time of passing one ion through the nanotube) in each SiC nanotubes. The retention time is shorter if the ions pass faster through the SiC nanotube, thus accelerating the separation process. The retention time was observed to decrease proportionally to the square root of the applied pressure. Furthermore, for large nanotubes the retention time was lower than for smaller nanotubes.
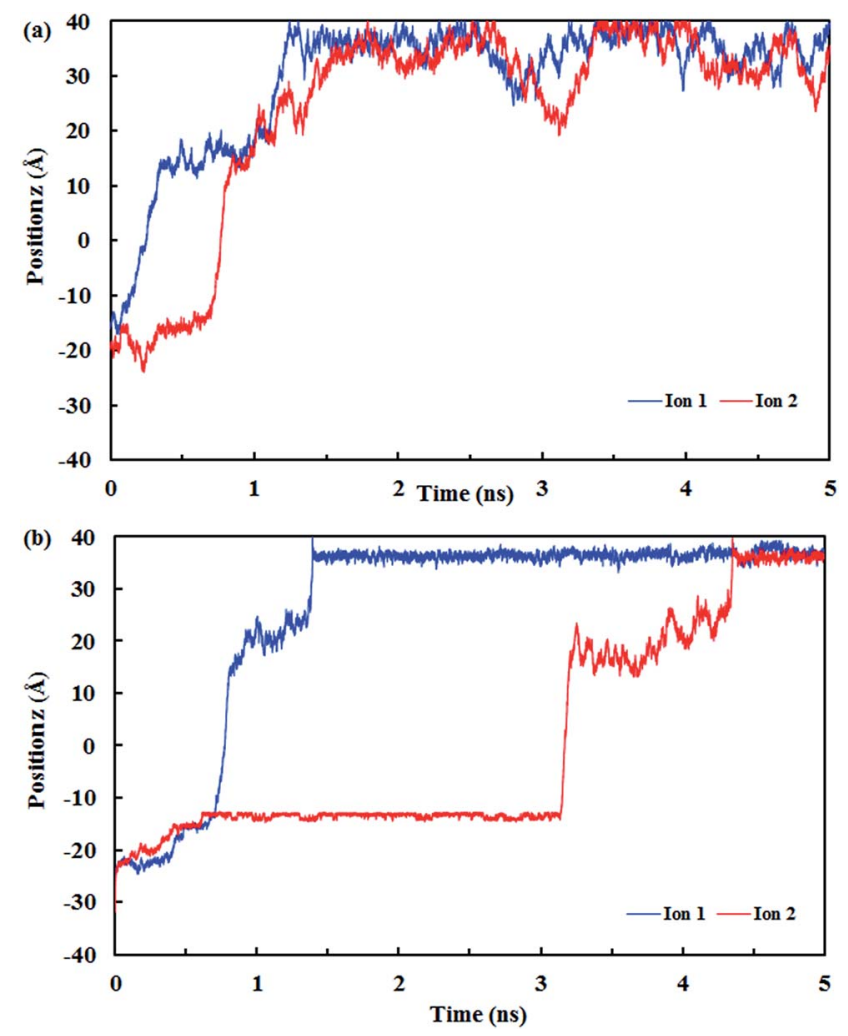

Fig. 10 The $z$ positions of the $\mathrm{CN}^{-}$ions at each simulation time at (a) low pressure (10 MPa) and (b) high pressure (150 MPa). 


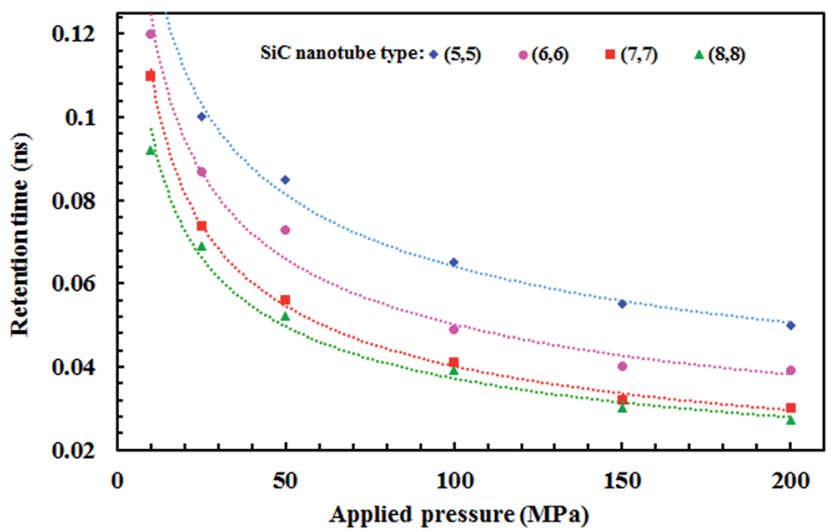

Fig. 11 Retention time of cyanide ions inside $\mathrm{SiC}$ nanotubes of different diameters.

\subsection{Radial distribution functions between cyanide ions and water molecules}

The RDF is defined as: ${ }^{57}$

$$
\mathrm{RDF}=\lim _{r \rightarrow 0} \frac{p(r)}{4 \pi \rho r^{2} \mathrm{~d} r}
$$

where $r$ and $\rho$ are the distance from the centre of the ion and the number density of water molecules in the system, respectively, and $p(r)$ is the number of water molecules around the ions found at a specific distance. This parameter can explain the
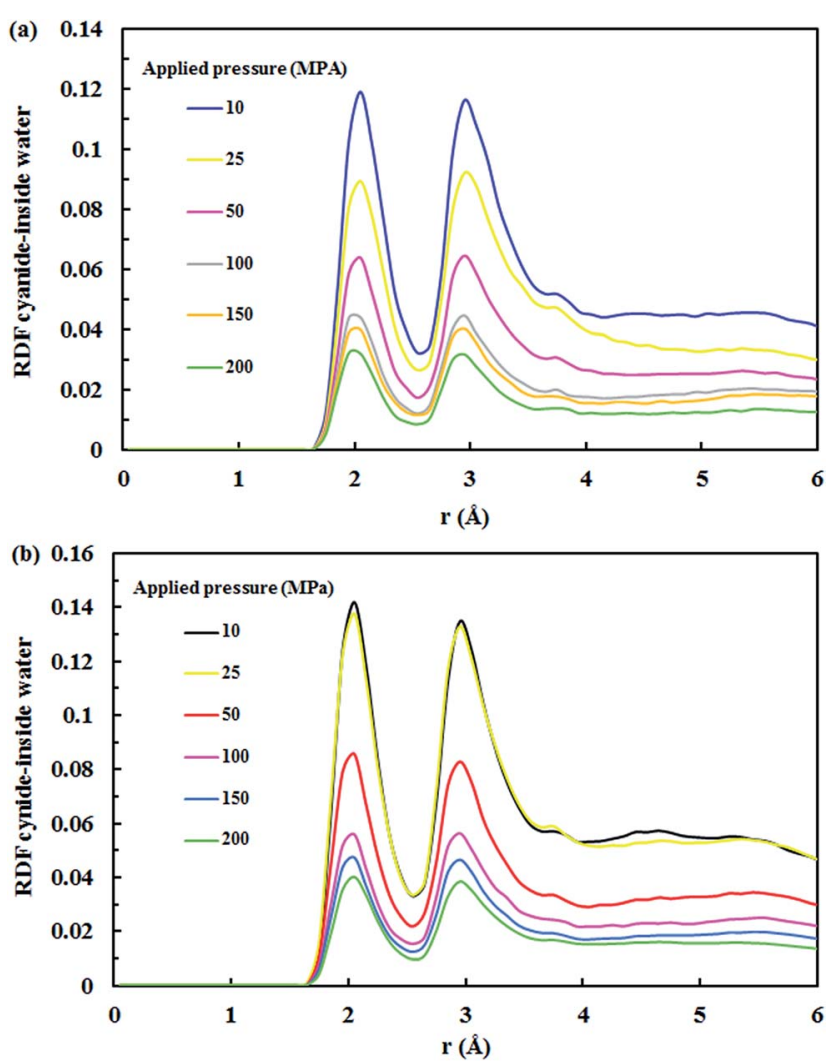

Fig. 12 RDF for cyanide ions-water at various applied pressures in the (a) $(7,7) \mathrm{SiC}$ nanotube and (b) $(8,8) \mathrm{SiC}$ nanotube. spatial density of water molecules around a specific ion. ${ }^{58}$ Fig. 12a and b show the cyanide-water RDF inside the $(7,7) \mathrm{SiC}$ nanotube and $(8,8) \mathrm{SiC}$ nanotube, respectively, both exhibiting two peaks. These two peaks represent the two hydration shells of the cyanide ion. It was observed that at short distances the RDF was zero, which was attributed to the repulsive forces between the cyanide ion centre and the water molecules. In all cases, the positions of the first peak and valley were the same. However, the heights were dissimilar for each pressure. This shows that the number of water molecules in the first hydration shell of the ion changed with a change in pressure. The heights of the first and second RDF peaks were almost equal in both the $(7,7)$ and $(8,8) \mathrm{SiC}$ nanotubes, and therefore, the amount of water molecules in the first and second hydration shell of the ion was the same. Furthermore, RDFs were altered by pressure. At low pressures, cyanide ions remained for a longer time inside the nanotubes, causing their RDF to intensify.

\section{Conclusion}

The separation of cyanide ions from aqueous solution with $\mathrm{SiC}$ nanotubes was studied via MD simulations, and $(5,5),(6,6),(7,7)$ and $(8,8) \mathrm{SiC}$ nanotubes were used. The results showed that a high rejection was achieved by $(5,5) \mathrm{SiC}$ nanotubes, which was $100 \%$ at $10 \mathrm{MPa}$. This rejection ability decreased when both the diameter of SiC nanotubes and the applied pressures increased. Moreover, it was found that the four types of SiC nanotubes accepted water molecules into their interior. The transportation of water molecules increased in the large nanotubes when applying high pressures, which compared with the theoretical HP equation. The large deviation of the MD result and macroscopic HP equation was a result of the inability of the HP equation for calculating fluid flows at a nanoscale.

\section{Acknowledgements}

Authors thank the University of Tabriz for the support provided. We also acknowledge the support of the Iran Science Elites Federation.

\section{References}

1 H. Simsek, M. Kobya, E. Khan and A. N. Bezbaruah, Environ. Technol., 2015, 36, 1612-1622.

2 C. A. P. Arellano and S. S. Martínez, Int. J. Hydrogen Energy, 2007, 32, 3163-3169.

3 M. Goncalves, A. Pinto and M. Granato, Environ. Technol., 1998, 19, 133-142.

4 T. Mudder and M. Botz, Eur. J. Miner. Process. Environ. Prot., 2004, 4, 62-74.

5 M. A. Acheampong, R. J. Meulepas and P. N. Lens, J. Chem. Technol. Biotechnol., 2010, 85, 590-613.

6 K. Baek, B.-K. Kim, H.-J. Cho and J.-W. Yang, J. Hazard. Mater., 2003, 99, 303-311.

7 R. R. Dash, C. Balomajumder and A. Kumar, Chem. Eng. J., 2009, 146, 408-413. 
8 S. Sirianuntapiboon and C. Chuamkaew, Bioresour. Technol., 2007, 98, 266-272.

9 G. Hassani, S. Nasseri and H. Gharibi, Anal. Bioanal. Electrochem., 2011, 3, 625-634.

10 R. Roshan Dash, C. Balomajumder and A. Kumar, Eng. Life Sci., 2009, 9, 53-59.

11 M. Hijosa-Valsero, R. Molina, H. Schikora, M. Müller and J. M. Bayona, Water Res., 2013, 47, 1701-1707.

12 M. Sarla, M. Pandit, D. Tyagi and J. Kapoor, J. Hazard. Mater., 2004, 116, 49-56.

13 S. Chergui, A. R. Yeddou, A. Chergui, F. Halet, H. Amaouche, B. Nadjemi and A. Ould-Dris, Toxicol. Environ. Chem., 2015, 97, 1289-1295.

14 A. Akcil, A. Karahan, H. Ciftci and O. Sagdic, Miner. Eng., 2003, 16, 643-649.

15 K. K. Ibrahim, M. A. Syed, M. Y. Shukor and S. A. Ahmad, BIOTROPIA, 2016, 22, 151-163.

16 S. C. Cheng, M. Gattrell, T. Guena and B. MacDougall, Electrochim. Acta, 2002, 47, 3245-3256.

17 T. Humplik, J. Lee, S. O'Hern, B. Fellman, M. Baig, S. Hassan, M. Atieh, F. Rahman, T. Laoui and R. Karnik, Nanotechnology, 2011, 22, 292001.

18 J. Theron, J. Walker and T. Cloete, Crit. Rev. Microbiol., 2008, 34, 43-69.

19 T. A. Hilder, D. Gordon and S.-H. Chung, Small, 2009, 5, 2183-2190.

20 Y. M. Kim, H. Ebro and J. H. Kim, Desalin. Water Treat., 2016, 1-8.

21 T. A. Hilder, R. Yang, D. Gordon, A. P. Rendell and S.-H. Chung, J. Phys. Chem. C, 2012, 116, 4465-4470.

22 P. S. Goh, A. F. Ismail and B. C. Ng, Desalination, 2013, 308, 2-14.

23 T. Bora and J. Dutta, J. Nanosci. Nanotechnol., 2014, 14, 613-626.

24 N. G. Chopra, R. Luyken, K. Cherrey, V. H. Crespi, M. L. Cohen, S. G. Louie and A. Zettl, Science, 1995, 269, 966-967.

25 L. Li, C. P. Li and Y. Chen, Phys. E, 2008, 40, 2513-2516.

26 D. Golberg, Y. Bando, W. Han, K. Kurashima and T. Sato, Chem. Phys. Lett., 1999, 308, 337-342.

27 X.-H. Sun, C.-P. Li, W.-K. Wong, N.-B. Wong, C.-S. Lee, S.-T. Lee and B.-K. Teo, J. Am. Chem. Soc., 2002, 124, 14464-14471.

28 C. Pham-Huu, N. Keller, G. Ehret and M. J. Ledoux, J. Catal., 2001, 200, 400-410.

29 R. Q. Wu, M. Yang, Y. H. Lu, Y. P. Feng, Z. G. Huang and Q. Y. Wu, J. Phys. Chem. C, 2008, 112, 15985-15988.

30 G. Mpourmpakis, G. E. Froudakis, G. P. Lithoxoos and J. Samios, Nano Lett., 2006, 6, 1581-1583.

31 J.-X. Zhao, B. Xiao and Y.-H. Ding, J. Phys. Chem. C, 2009, 113, 16736-16740.

32 A. Khataee, J. Azamat and G. Bayat, Comput. Mater. Sci., 2016, 119, 74-81.

33 R. Saghatchi, J. Ghazanfarian and M. Gorji-Bandpy, J. Offshore Mech. Arct. Eng., 2014, 136, 031801.

34 A. Yazdani, M. Deng, B. Caswell and G. E. Karniadakis, J. Comput. Phys., 2016, 305, 906-920.
35 H. J. Berendsen, Simulating the physical world: hierarchical modeling from quantum mechanics to fluid dynamics, Cambridge University Press, 2007.

36 N. Modi, M. Winterhalter and U. Kleinekathoefer, Nanoscale, 2012, 4, 6166-6180.

37 M. Thomas, B. Corry and T. A. Hilder, Small, 2014, 10, 1453-1465.

38 T. Darden, D. York and L. Pedersen, J. Chem. Phys., 1993, 98, 10089-10092.

39 M. W. Schmidt, K. K. Baldridge, J. A. Boatz, S. T. Elbert, M. S. Gordon, J. H. Jensen, S. Koseki, N. Matsunaga, K. A. Nguyen and S. Su, J. Comput. Chem., 1993, 14, 1347-1363. 40 M. Menon, E. Richter, A. Mavrandonakis, G. Froudakis and A. N. Andriotis, Phys. Rev. B: Condens. Matter, 2004, 69, 115322.

41 Y. Zhang and H. Huang, Comput. Mater. Sci., 2008, 43, 664-669. 42 W. L. Jorgensen, J. Chandrasekhar, J. D. Madura, R. W. Impey and M. L. Klein, J. Chem. Phys., 1983, 79, 926-935.

43 J. C. Phillips, R. Braun, W. Wang, J. Gumbart, E. Tajkhorshid, E. Villa, C. Chipot, R. D. Skeel, L. Kale and K. Schulten, J. Comput. Chem., 2005, 26, 1781-1802.

44 J. Azamat, A. Khataee and S. W. Joo, Chem. Eng. Sci., 2015, 127, 285-292.

45 J. Azamat, A. Khataee and S. W. Joo, J. Mol. Struct., 2016, 1108, 144-149.

46 J. Azamat, A. Khataee and S. W. Joo, J. Mol. Model., 2016, 22, 1-8.

47 J. Azamat, J. Phys. Chem. C, 2016, 120, 23883-23891.

48 W. Humphrey, A. Dalke and K. Schulten, J. Mol. Graphics, 1996, 14, 33-38.

49 J. Azamat, J. J. Sardroodi and A. Rastkar, Desalin. Water Treat., 2015, 56, 1090-1098.

50 B. J. Bucior, D.-L. Chen, J. Liu and J. K. Johnson, J. Phys. Chem. C, 2012, 116, 25904-25910.

51 J. Azamat, A. Khataee and S. W. Joo, J. Mol. Model., 2014, 20, 2468.

52 F. Zhu, E. Tajkhorshid and K. Schulten, Biophys. J., 2002, 83, 154-160.

53 F. Zhu, E. Tajkhorshid and K. Schulten, Biophys. J., 2004, 86, 50-57.

54 D. F. Young, B. R. Munson, T. H. Okiishi and W. W. Huebsch, A brief introduction to fluid mechanics, John Wiley \& Sons, 2010.

55 R. Saghatchi and J. Ghazanfarian, Applied Mathematical Modelling, 2015, 39, 1063-1073.

56 Y. A. Cengel and M. A. Boles, Sea, 1994, 1000, 8862.

57 A. Kalinichev and R. Kirkpatrick, Eur. J. Soil Sci., 2007, 58, 909-917.

58 A. A. Dezfoli, M. Mehrabian and H. Hashemipour, Chem. Eng. Commun., 2015, 202, 1685-1692.

59 F. Taghavi, S. Javadian and S. M. Hashemianzadeh, J. Mol. Graphics Modell., 2013, 44, 33-43.

60 D. Cohen-Tanugi and J. C. Grossman, Nano Lett., 2012, 12, 3602-3608.

61 A. D. MacKerell Jr, D. Bashford, M. Bellott, R. L. Dunbrack Jr, J. D. Evanseck, M. J. Field, S. Fischer, J. Gao, H. Guo and S. Ha, J. Phys. Chem. B, 1998, 102, 3586-3616.

62 M. W. Lee and M. Meuwly, J. Phys. Chem. A, 2011, 115, 50535061. 